\title{
Manejo prehospitalario de la hipotermia accidental; Una emergencia sanitaria en el Mediterráneo
}

\author{
Itziar Ibáñez Grima ${ }^{1}{ }^{*}$, Iván Ortega Deballón ${ }^{2}$ \\ 1 Graduada en Enfermería, Hospital Universitario Miguel Servet, Máster Universitario en Acción \\ Humanitaria Sanitaria; ORCID id: https://orcid.org/0000-0002-6995-1926 \\ 2 Enfermero de urgencias, Profesor asociado de la Facultad de Medicina y Ciencias de la Salud, Universidad \\ Alcalá: ORCID id: https://orcid.org/0000-0002-0819-2399 \\ * Autor correspondencia: itziar992@gmail.com
}

DOI: https://doi.org/10.37536/RIECS.2020.5.S1.174

Recibido: 30/01/2020; Aceptado: 05/02/2020; Publicado: 14/02/2020

Resumen: 1) Antecedentes: Las diferentes rutas migratorias que atraviesan el Mar Mediterráneo son consideradas las rutas más mortíferas de 2019. Son varios los organismos que recopilan información sobre estos movimientos migratorios, refugiados y migrantes deben ser asistidos cuando se encuentren en peligro según el Derecho Marítimo y trasladados a un puerto seguro en el cual se respeten sus Derechos Humanos. La OMS resalta que la hipotermia se sitúa entre los problemas de salud más frecuentes en el Mediterráneo seguido de quemaduras y lesiones accidentales. Las muertes por ahogamiento e hipotermia representan más del $50 \%$ de los casos según la OIM convirtiendo esta patología en una emergencia sanitaria cuyo abordaje prehospitalario definirá los resultados clínico-asistenciales. 2) Métodos: Revisión sistemática de tipo exploratoria para describir el conocimiento sanitario actual sobre la hipotermia accidental tras inmersión y como el correcto triaje, clasificación del grado de hipotermia y tratamiento influyen en los resultados clínicoasistenciales prehospitalarios. 3) Resultados: El triaje debe ser llevado a cabo por profesionales experimentados en el ámbito marítimo. El tratamiento recibido debe ser acorde al grado de hipotermia que presente el paciente, el cuál será definido por la clínica. Los casos de HA severa y PCR por hipotermia cumplen los criterios para una derivación hospitalaria urgente. 4) Conclusión: La hipotermia accidental es una patología ambiental, se considera una emergencia y tiene unos principios básicos de clasificación, tratamiento y reanimación. Conocer estos principios asegurará un buen manejo prehospitalario del paciente hipotérmico.

Palabras Clave: Hipotermia accidental, Inmersión, Triaje, Tratamiento Prehospitalario, Mediterráneo, Migrantes.

Abstract: 1) History: The various migrant routes that cross the Mediterranean Sea are deemed to be the deadliest in 2019. They are several agencies that collect data about these migratory flows; refugees and migrants must be assisted when they are in danger according to Maritime Law and transferred to a safe port whereby their human rights must be respected. The WHO highlights that hypothermia is placed into most frequent health risks in the Mediterranean Sea, followed by burns and accidental injuries. Deaths by drowning and hypothermia depict more than $50 \%$ of the cases; which according to the WHO is turning this pathology into a health emergency whose pre-hospital approach will determine the clinical-care results. 2) Methodology: An exploratory systematic review in order to represent the actual health knowledge about accidental hypothermia after immersion and how an accurate triage, classification of hypothermia grade and treatment can impact in pre-hospital clinical-care results. 3) Outcomes: Triage has to be accomplished by seafaring field experienced professionals. The medical treatment received must be in keeping with hypothermia scale that the patient has, which will be specified by clinic. Severe accidental hypothermia and cardiac arrest due to hypothermia both meet the criteria for an urgent hospital referral. 4) Conclusion: Accidental hypothermia is an environmental pathology; it is considered an 
emergency and has its own key rules for classification, treatment and resuscitation. Understanding these principles will ensure a correct pre-hospital operation of hypothermic patient.

Key words: Accidental Hypothermia, Immersion, Triage, Prehospital Treatment, Mediterranean Sea, Migrants.

\section{Introducción}

\subsection{Antecedentes, legislación y problemática actual}

En los últimos años, el Mediterráneo se ha convertido en un punto estratégico de los movimientos migratorios ya que en él confluyen: zonas de emisión de emigración, zonas de recepción y zonas de tránsito [1].

En 2016, fueron más de 5.000 las personas que perdieron la vida en el Mediterráneo. Está considerado el año más trágico por varias ONGs, entre las cuales destaca ACNUR que atribuye el elevado número de muertes a unas embarcaciones de peor calidad, condiciones meteorológicas adversas en el Mediterráneo central y el envío simultáneo de varias embarcaciones por parte de los traficantes, en su conjunto dificultaron el trabajo de los equipos de rescate [2].

Aunque el número de muertes está disminuyendo ACNUR advierte que se debe realizar una doble lectura de los datos, ya que la reducción de capacidad de búsqueda y salvamento, y la descoordinación e incertidumbre sobre el desembarco de las personas rescatadas son factores que pueden estar sesgando las cifras [3,4].

Según la OIM, el $48 \%$ de las personas muertas o desaparecidas durante un trayecto migratorio hacia un destino internacional en 2018 lo hicieron en el Mediterráneo [5].

La obligación de asistencia a las personas que se encuentren en peligro en el mar es un principio esencial y básico del Derecho Marítimo. Además, está reconocido en la legislación internacional destacando: el artículo 38 de la Convención de las Naciones Unidas sobre el derecho del Mar, la Regla 33 del capítulo V del Convenio Internacional para la Seguridad de la Vida Humana en el Mar (SOLAS) y el artículo 2.1.10 del Convenio internacional sobre Búsqueda y Salvamento (SAR). [6-8]

El Estatuto del Refugiado de 1951 en su artículo 31 establece que los estados no pueden sancionar a las personas que, de manera ilegal, entren en su territorio si su vida o libertad está amenazada en su lugar de procedencia. No se les debe tratar con discriminación alguna y mucho menos devolverlos al lugar de origen donde pueden sufrir persecución haciendo valer el principio de non-refoulement [9].

Se puede considerar que en el Mediterráneo no sólo se da una gran crisis migratoria y de refugiados, sino que ha evolucionado en una gran crisis humanitaria debido a la reducción de las operaciones de búsqueda y salvamento, la cooperación con países que no respetan los derechos humanos y la inexistencia de rutas seguras y legales para evitar que las personas que huyen deban aventurarse en la ruta considerada la más letal por varias ONGs $[2,10,11]$

Se entiende que las ONGs, organizaciones sin ánimo de lucro creadas por la sociedad civil con el objeto de crear un determinado impacto en la sociedad, defienden y representan mediante sus actividades los principios éticos y operativos, entre los que destacan: la humanidad, imparcialidad, neutralidad, independencia y universalidad, los cuales definen la base de la acción humanitaria [12].

Debido a la necesidad de hacer respetar los derechos humanos de los migrantes y refugiados que intentan llegar a Europa a través del Mediterráneo varias las ONGs patrullan el mar en busca de personas a la deriva que precisen ser recatadas, entre los barcos de rescate más conocidos se encuentran: Sea Watch 3, Open Arms y Ocean Viking [13-16].

A pesar de este hecho, las muertes en el Mediterráneo por ahogamiento e hipotermia representan más del 50\% de los casos según las estadísticas del proyecto "Missing Migrants" de la OIM [5]. 
La OMS concluye que la hipotermia se sitúa entre los problemas de salud más frecuentes en migrantes y refugiados, además de lesiones accidentales, quemaduras, accidentes cardiovasculares, shock, complicaciones del embarazo y parto, así como la descompensación de enfermedades crónicas como diabetes o hipertensión. La hipotermia es una constante en las emergencias del Mediterráneo [17].

Se define la hipotermia accidental cuando la temperatura corporal central (TCC) se encuentra por debajo de $\operatorname{los} 35^{\circ} \mathrm{C}$, ocurre de forma no intencionada, espontánea, sin una lesión previa en el hipotálamo y en un ambiente frío (como es la inmersión en agua fría) [18].

\subsection{Objetivos}

- Evaluar el conocimiento actual sanitario sobre la hipotermia accidental tras inmersión como emergencia sanitaria en el contexto marítimo.

- Identificar los métodos más idóneos para un realizar un triaje de calidad en un contexto extrahospitalario marítimo en el que se debe socorrer a varias personas.

- Identificar correctamente los diferentes grados de hipotermia, su tratamiento extrahospitalario, así como los criterios de derivación hospitalaria urgente.

\section{Material y Métodos}

\subsection{Tipo de estudio y formulación de la pregunta de investigación}

Se realizó una revisión sistemática de tipo exploratoria para describir el conocimiento sanitario actual sobre la hipotermia accidental tras inmersión y como el correcto triaje, clasificación del grado de hipotermia y tratamiento influyen en los resultados clínicoasistenciales prehospitalarios.

Para responder al objetivo del estudio se formuló una pregunta de investigación siguiendo la nemotecnia PICO. La pregunta en cuestión quedó formulada de la siguiente manera: ¿las personas que padecen hipotermia accidental que son correctamente triadas, clasificadas y tratadas según el grado de hipotermia que padezcan presentan menor probabilidad de muerte que aquellas que no?

\subsection{Criterios de inclusion y exclusión}

Respecto al documento: Año de publicación: 2014-2019. Idioma: textos en español e inglés. Ámbito geográfico: mundial. Respecto al contenido del artículo: todo tipo.

\subsection{Identificación de las fuentes de información y estrategias de búsqueda}

Se realizó una búsqueda en las siguientes bases de datos biomédicas: Pubmed (medline), Scielo, Dialnet, Cocrhane; y en literatura gris entre septiembre y diciembre de 2019. Fueron utilizados los descriptores específicos de cada base de datos MeSH y DeCs para obtener la colección de artículos adecuada de los estructurados en el Thesauro para trabajar con lenguaje controlado y lograr la mayor especificidad y sensibilidad en la búsqueda, a través de replicar la estrategia de búsqueda utilizada para clasificar los artículos en el momento que fueron introducidos en la base de datos. También fueron activados sistemas de alerta para la búsqueda actualizada con los nuevos documentos publicados.

\subsection{Selección y clasificación de los artículos}

- Primer filtro: Aplicación de los criterios de inclusión y exclusión, y en los estudios que superen los criterios de inclusión se revisa el título y resumen del artículo. Se clasifica como "pertinente" o "no pertinente" para descartar aquellos artículos que no se adaptasen a la pregunta de investigación. 
- Segundo filtro: Se revisan los textos completos. Los estudios son valorados a través del software FLC (fichas de lectura crítica) desarrollado por el Servicio Vasco de Evaluación de Tecnologías Sanitarias (OSTEBA), el cual permite valorar la calidad de los estudios según el rigor metodológico en alta, media, baja y no valorable.

\section{Resultados}

La búsqueda bibliográfica identificó 1.247 artículos de potencial interés. De ellos, 265 fueron seleccionados como pertinentes según el modelo propuesto aplicando los criterios de inclusión y exclusión. En un segundo filtro revisando textos completos, y realizando un análisis exhaustivo de la información, en el cual se evaluó la utilidad y calidad, 38 artículos fueron elegidos para ser incluidos finalmente en la revisión.

La evaluación de la calidad de los artículos se realizó a través de la lectura crítica a través de las fichas OSTEBA que permitió jerarquizar la evidencia científica en función del rigor metodológico, incluyéndose en esta revisión aquellos estudios que arrojaron un nivel de calidad medio, alto y no valorable (como es el caso de las revisiones bibliográficas).

De los artículos seleccionados, el 60,6\% fueron obtenidos de Pubmed, el 13,2\% de Cocrhane, el 5,2\% de Dialnet, el 5,2\% de Scielo y el 15,8\% restante de literatura gris como Google académico y Guidelines como se muestra en la Figura 1, perteneciendo el 86,8\% de los artículos incluidos al intervalo 2014-2019 y siendo el 76,3\% del total artículos textos en inglés frente al $23,7 \%$ de habla hispana.

Fuentes de los estudios incluidas en esta revisión

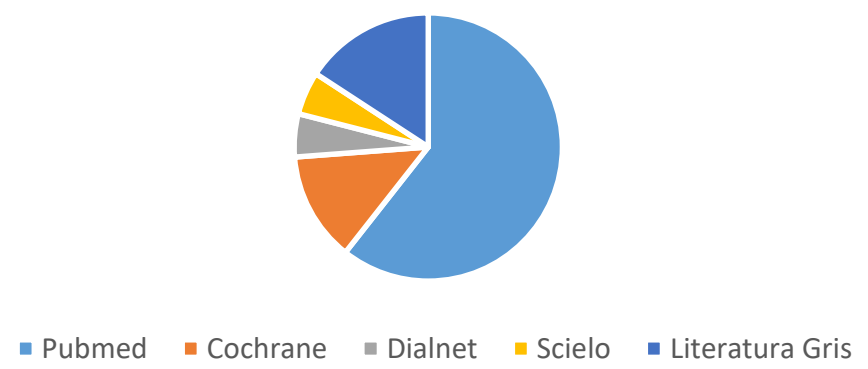

Figura 1 Fuentes de los estudios incluidas en esta revisión. Elaboración propia.

Entre estos estudios se encuentran diferentes tipos de estudios epidemiológicos, tanto analíticos como descriptivos como se puede ver en la Figura 2.

\section{TIPOS DE ESTUDIOS INCLUIDOS EN ESTA REVISIÓN}

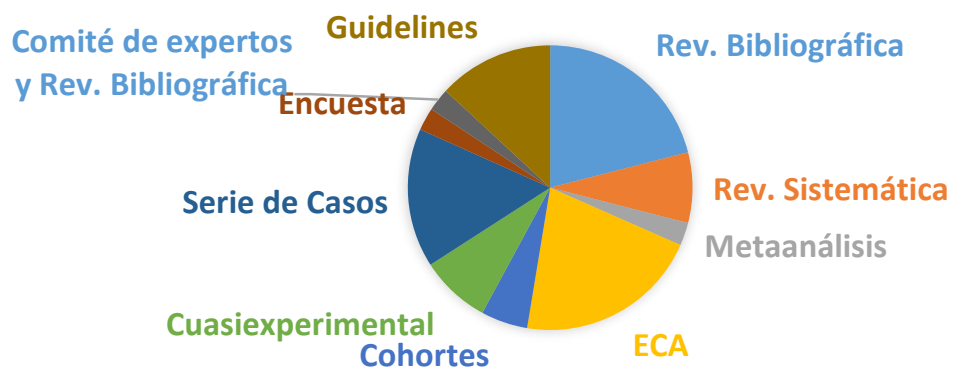

Figura 2 Tipos de estudios incluidos en esta revisión. Elaboración propia. 


\section{Discusión}

\subsection{Triaje}

Todos los estudios revisados inciden en la importancia de realizar un buen triaje para reducir la morbimortalidad posterior y reducir los tiempos de traslado [20-24].

El medio marítimo es un medio de gran dificultad para obtener datos y poder proporcionar una asistencia sanitaria de calidad, cuando los servicios de rescate llegan hasta las personas que precisan ayuda $[19,20]$ la prioridad es garantizar la seguridad del rescatador [31].

Una vez el escenario sea seguro para realizar el rescate se proporcionarán elementos de flotabilidad siguiendo a cadena de supervivencia ante el ahogamiento y se priorizará el rescate de aquellas personas que se encuentren próximas a la inmersión en menos de 1 minuto, seguidas de aquellas personas que presenten ansiedad, pero que colaboran en su rescate, después las personas que permanecen tranquilas y colaboradoras y en último lugar aquellas personas que no responden y tienen el cuerpo y la cara ya sumergidos en el agua. Aplicando de esta manera el método de triaje START acuático [21].

Una vez ya en el barco de rescate se deberá realizar un nuevo triaje según el cual se iniciará el mejor tratamiento disponible en función de las necesidades clínico-asistenciales.

Aunque el método de triaje START está ampliamente reconocido y con él se obtienen buenos resultados, el método de triaje META, utilizado sistemáticamente por sanitarios experimentados en su uso, podría reducir significativamente los tiempos de evacuación [22]. Otro de los factores que disminuiría el tiempo de actuación y evacuación sería la actuación personal sanitario con gran experiencia en el ámbito de la emergencia, en este caso el ámbito marítimo, favoreciendo un triaje más intuitivo [23].

La posibilidad de realizar un triaje por medio de tecnología de vehículos aéreos no tripulados (drones) frente al triaje habitual facilitaría la preevaluación de las víctimas antes de la llegada física el equipo de rescate, algo que sería de gran utilidad emergencias marítimas [24].

No cabe duda de que la utilización de drones como herramienta de apoyo sanitario supone un gran avance, pero ¿cómo poder encontrar a posibles náufragos en mitad del Mediterráneo en plena noche?

Desde hace tiempo, los helicópteros de Salvamento Marítimo disponen de cámaras infrarrojas para la detección de personas vivas en el mar, éstas cámaras permiten a los operadores del helicóptero rastrear una zona en busca de supervivientes. Sin embargo, es un sistema con ciertas limitaciones como son: rastreo a baja altitud y velocidad por lo que se cubre poca superficie por horas y la dependencia del cansancio, la agilidad y agudeza visual del operador.

El Proyecto Picasso, proyecto europeo liderado por SASEMAR, ha logrado desarrollar un sistema automático de detección de personas mediante un algoritmo de discriminación de objetivos para lograr de este modo una detección desde mayor altitud y velocidad. A nivel mundial, se trata de un sistema completamente novedoso ya que el algoritmo se ha podido desarrollar gracias a un dispositivo con la capacidad de reproducir la temperatura facial y craneal de un náufrago vivo en diferentes condiciones, dicho dispositivo ha resultado clave para el diseño del algoritmo de búsqueda y detección permitiendo diferenciar lo que debía identificar como objetivo de lo que tenía que ignorar por ser un falso objetivo. La experiencia piloto terminó en 2018 y todavía se sigue trabajando para llevar este proyecto a la realidad [25].

\subsection{Medición de la TCC}

Para un correcto diagnóstico de hipotermia la TCC debe encontrarse por debajo de $35^{\circ} \mathrm{C}$, la recomendación para realizar la medición de forma adecuada y precisa de la TCC es a través de una sonda esofágica [18,31,38]. No obstante, pocas son las emergencias extrahospitalarias en las que se dispone de dicha sonda siendo aceptada la medición de la TCC de manera timpánica, aunque presenta menor fiabilidad en ambientes fríos o si hay agua en el conducto auditivo [18,44]. 
Según la Guía Sanitaria a Bordo del ISM, todos los barcos deben llevar en su botiquín un termómetro digital que se localiza en el cajón 11 de los botiquines tipo A, B y C (el tipo de botiquín varía según la distancia de la costa a la que están autorizados a realizar su actividad, siendo el botiquín tipo A el más completo y el C el más básico)[32] sin embargo, resulta llamativo el hecho de que no se ha encontrado constancia de la presencia en estos botiquines de un termómetro timpánico, sino de termómetros digitales y/o de mercurio.

Debido a estas dificultades a la hora de medir la TCC en el medio extrahospitalario, la AHA, el ERC y otros autores recomiendan adecuar el tratamiento a la clínica que presente el paciente hipotérmico si no se disponen de los mecanismos adecuados para realizar la medición, sabiendo que la clínica está directamente relacionada con la TCC [18,27,31,32,34,35].

\subsection{Técnicas de recalentamiento}

Los estudios revisados muestran una clara evidencia de que las técnicas de recalentamiento son un pilar fundamental en el tratamiento de la hipotermia [29-35, 42].

La técnica de elección para el tratamiento de la hipotermia leve es el recalentamiento pasivo externo, que consiste en aislar al paciente y proporcionarle un ambiente cálido por medio de mantas [29-31,33-35], el uso de mantas diseñadas específicamente para proporcionar un buen aislamiento y evitar la formación de vapor es habitual en el $85 \%$ de los rescates marítimos en pacientes con este diagnóstico [44]. Estas medidas son aplicables en el entorno extrahospitalario.

En la memoria de 2017 de la ONG Proactiva Open Arms, en el punto 5 enfocado a las operaciones de rescate, se indica como la tripulación además de identificar a las personas procedentes de cada embarcación, deben proporcionarles ropa seca a las personas que así lo precisen además de una manta individual para cada una [14]. Entendiéndose de este modo la correcta actuación sanitaria de ésta ONG al tratar y evitar que aumente el grado de hipotermia en los migrantes y refugiados rescatados por este barco.

No obstante, hay evidencia de que incluso en hipotermias leves estaría recomendado, el uso de técnicas de recalentamiento activo externo (mantas eléctricas, inmersión de extremidades en agua tibia, parches de calor, etc) como técnica complementaria y no como tratamiento exclusivo de hipotermias moderadas y severas [29,31,33,43,55].

En la Guía Sanitaria a Bordo se especifica que es recomendable además de intentar calentar al paciente con mantas, añadir calor gradual lentamente especialmente en cabeza, cuello (si se puede evitar la pérdida de calor a través de la abertura de la manta en dicha zona) [31], pecho e inglés mediante almohadas o botellas de agua caliente en náufragos con temblores, es decir, con hipotermia leve [18,32]. Esta recomendación se basa en que la combinación de estos dos métodos de recalentamiento produce una disminución de los temblores, disminución segura del tiempo de recalentamiento y, por ende, el aumento de confort referido por el paciente $[31,43,51,53,55]$.

En hipotermias moderadas o graves el tratamiento de elección es el recalentamiento activo externo [29]. Con este método de recalentamiento se puede incluso duplicar la tasa de recalentamiento frente a métodos pasivos externos [46,53].

Varios de los estudios revisados se centran específicamente en el recalentamiento activo externo y determinan que la combinación de varios métodos activos externos resulta beneficiosa para el paciente disminuyendo de manera segura el tiempo de recalentamiento $[43,51,52,55]$. Solo dos de los estudios se centran en una combinación concreta que es la utilización de una manta eléctrica junto con un sistema de aire forzado, cuyos resultados son óptimos en relación con la tasa de recalentamiento y el confort del paciente [52,55].

Por otro lado, también se han encontrado estudios de carácter comparativo en el que se intenta determinar que método de recalentamiento activo externo es más efectivo de manera individual.

La inmersión de las extremidades distales en agua, técnica también denominada shunt arteriovenoso [18], está recomendada en pacientes con hipotermia leve en un entorno clínico adecuado siempre y cuando el agua este entre $42-45^{\circ} \mathrm{C}$ [31], resulta más efectiva en comparación con los parches de calor seco,68 lo que no quiere decir que estos parches no sean útiles ya que la 
colocación de estos parches en las anastomosis arteriovenosas (como son las palmas de las manos y plantas de los pies) reduce los temblores, en combinación con otros métodos lo que se traduce en un mayor confort del paciente, aunque no hay evidencia de que mejore el tiempo de recalentamiento de forma aislada [51].

Los métodos de recalentamiento activo externo son métodos seguros si se utilizan adecuadamente [43,46,48,51-53,55], de no ser así pueden producir lesiones y provocar el efecto afterdrop consecuencia de una reperfusión y reoxigenación inadecuadas [50].

Para evitar las complicaciones derivadas de un recalentamiento incorrecto, el personal sanitario debe tomar las medidas oportunas para evitar posibles quemaduras de contacto [18,31] entre las que se encuentran: seguir las instrucciones del fabricante y controlar la zona en contacto con la fuente de calor la cual debe estar protegida mediante una capa aislante de modo que no haya un contacto directo sobre la piel [47].

La evidencia revisada concluye que el recalentamiento extrahospitalario debe comenzar lo antes posible y debe ser lento ya que estos dos factores están directamente relacionados con el aumento de la tasa de supervivencia y la disminución del efecto afterdrop [41]. Se entiende por recalentamiento lento el aumento aproximado de la TCC de unos $2^{\circ} \mathrm{C} / \mathrm{h}$ en hipotermias leves y moderadas $[31,45,51]$ con métodos de recalentamiento activos externos y entre $0.1-0.7^{\circ} \mathrm{C} / \mathrm{h}$ con técnicas de recalentamiento pasivo externo. Ambos tipos son aconsejables a nivel extrahospitalario [18] como podría ser un barco de rescate.

Los métodos de recalentamiento activos internos a nivel prehospitalario como son los gases humidificados o los líquidos calientes intravenosos no son eficientes [18]. Sin embargo, pueden ser de gran utilidad durante el traslado del paciente. El oxígeno calentado y humidificado debe emplearse siempre en combinación con otros métodos [31] y la fluidoterapia intravenosa para que sea efectiva debe realizarse con fluidos salinos a $42^{\circ} \mathrm{C}$ algo difícil de lograr en entornos no hospitalarios donde los sueros se encuentran a temperatura ambiente [51].

\subsection{Criterios de derivación hospitalaria}

Los estudios revisados determinan como criterio de derivación hospitalaria urgente al paciente con hipotermia severa o al paciente hipotérmico en PCR [27,34,35,37-39,42].

No obstante, las maniobras de resucitación deben iniciarse en el entorno extrahospitalario salvo si existen claros indicios de muerte en el paciente como: rigor mortis, decapitación, descomposición, etc. $[31,34,35]$.

Son múltiples los informes de casos de pacientes hipotérmicos sin signos vitales que sobrevivieron a RCP prolongada, incluso con tiempos de inactividad de RCP durante el traslado hacia un centro hospitalario [28,34-36]. En el caso de una RCP no continua o intermitente las compresiones se deben realizar durante al menos 5 minutos y las interrupciones no deberían superar este mismo tiempo, siendo el ritmo de las compresiones igual al empleado en pacientes normotérmicos [31].

En pacientes pediátricos hipotérmicos por inmersión y que han sufrido ahogamiento se considera un factor de mal pronóstico la no restauración de las funciones circulatorias de manera espontánea (ROSC) tras 30 minutos de RCP [40].

Otros factores de mal pronóstico relacionados con el aumento de la mortalidad en población adulta debido a hipotermia accidental son: edad avanzada, TCC muy baja y que la hipotermia sea consecuencia de un periodo de inmersión [49]. Como determinantes de un buen pronóstico neurológico en supervivientes tras tratamiento con ECLS se encuentran el sexo femenino, la no presencia de asfixia y cifras disminuidas de potasio sérico [39], además de la ausencia de hipoxia cerebral previa [29]. Esto se debe a que en la hipotermia por inmersión se reduce el consumo de oxígeno cuya consecuencia es un efecto preventivo sobre la hipoxia cerebral [18].

La bibliografía revisada incide en que un paciente hipotérmico no ha fallecido hasta que se ha completado el recalentamiento, debido al efecto protector que causa la hipotermia en el sistema neurológico [31,34-36,38]. 
El centro hospitalario debe contar con ECLS para aquellos pacientes que sean derivados por hipotermia severa inestable o PCR hipotérmica [31]. Por lo que se puede asumir la importancia de que los servicios de rescate y/o los centros de coordinación cuenten con un listado de hospitales que poseen ECLS ya que, de no ser así, se podrían realizar traslados innecesarios con un paciente en estado crítico [18].

Proporcionar ECLS a un paciente hipotérmico severo o en PCR por hipotermia no asegura su supervivencia. Las tasas de supervivencia y de recuperación neurológica como medidas de resultado difieren de un estudio a otro: Mwaura en su estudio determinó una supervivencia del $50 \%$ de los cuales un 90\% no padecía lesiones neurológicas [37], Saczkowski estimó un buen resultado neurológico en el 40,3\% de la cohorte que estudió [39], Avellanas concluyó que la tasa de supervivencia se encuentra en cifras cercanas al 100\% [42] y Paal indica que se trata de un método efectivo y que las tasas de supervivencia son variables (23-100\%), dicha variabilidad depende de la gran variedad de factores que influyen en el proceso hipotérmico: entorno, causas, estado basal, problemas durante el rescate y traslado, instalaciones hospitalarias y tratamientos disponibles [27].

La evidencia revisada coincide en que es el tratamiento de elección en los casos de hipotermia severa y PCR por las elevadas posibilidades de supervivencia y de buenos resultados neurológicos $[31,34-37,39,40,42]$.

Otro punto común de los estudios es la determinación del ECMO como uno de los métodos más utilizados en ECLS por sus buenos resultados en pacientes hipotérmicos $[18,29,31,36,54]$ o el uso del bypass cardiopulmonar (CPB) $[18,27,31]$. Se trata de métodos altamente invasivos capaces de elevar la TCC en $7-10^{\circ} \mathrm{C} / \mathrm{h}$ [29]. Se prefiere el ECMO frente al CPB en pacientes con PCR o hipotermia severa (con inestabilidad hemodinámica) [31] ya que proporciona de manera simultánea oxigenación, soporte circulatorio, recalentamiento y precisa menor anticoagulación [29]. Debido a estas ventajas se considera el ECMO como "Gold Standard" en la PCR hipotérmica [35].

\section{Conclusiones}

1. La hipotermia accidental se clasifica dentro de las patologías ambientales, se considera una emergencia y tiene unos principios básicos de clasificación, tratamiento y reanimación aplicables tanto en el medio marítimo como en el montañoso o el urbano.

2. En un naufragio en el que varias personas precisan rescate y asistencia sanitaria, se priorizará la seguridad del rescatador el cual deberá tener experiencia en este tipo de rescate. Se seguirá la cadena de supervivencia ante el ahogamiento y se realizará un triaje START acuático para realizar un rescate adecuado y ordenado.

3. El grado de hipotermia en el ámbito extrahospitalario será definido por la clínica del paciente

4. El tratamiento recibido será acorde al grado de hipotermia que presenta el paciente y éste tendrá como base la prevención de una mayor pérdida de calor y un recalentamiento precoz.

5. Un paciente en PCR por hipotermia o con hipotermia severa cumple los criterios para una derivación hospitalaria urgente. Además, un paciente en PCR debe recibir siempre RCP ya que, a diferencia de otras patologías, en la hipotermia no se debe considerar muerto al paciente hasta que no se haya concluido el periodo de calentamiento, generalmente en un centro hospitalario mediante técnicas de ECLS.

6. Las técnicas de ECLS, entre las que destaca el ECMO, son el tratamiento de elección en los casos de hipotermia severa y PCR por las elevadas posibilidades de supervivencia y de buenos resultados neurológicos. 
Agradecimientos: Me gustaría dar mi más sincero agradecimiento a todas las personas que me han acompañado, de una forma u otra, en el desarrollo de este Trabajo Fin de Máster con el que finaliza esta etapa de aprendizaje.

Contribución de los autores: He realizado la presente revisión como única revisora bajo la tutorización de mi tutor, Iván Ortega.

Conflictos de Intereses: Los autores no declaran conflicto de intereses.

\section{Abreviaturas}

Las siguientes abreviaturas son usadas en este manuscrito:

ACNUR: Agencia de la ONU para los Refugiados

AHA: American Heart Association

BCP: Bypass Cardiopulmonar

DIH: Derecho Internacional Humanitario

ECLS: Soporte Vital Extra Corpóreo

ECMO: Membrana de Oxigenación Extra Corpórea

ERC: European Resucitation Council

FLC: Fichas de Lectura Crítica

ISM: Instituto Social de la Marina

META: Modelo Extrahospitalario de Triaje Avanzado

OIM: Organización Internacional para las Migraciones

OMS: Organización Mundial de la Salud

ONG: Organización No Gubernamental

OSTEBA: Servicio Vasco de Evaluación de Tecnologías Sanitarias

PCR: Parada Cardiorrespiratoria

RCP: Reanimación Cardiopulmonar

ROSC: Restauración de las Funciones Circulatorias de Manera Espontánea

SAR: Búsqueda y Salvamento

SASEMAR: Sociedad de Salvamento y Seguridad Marítima

START: Triaje Simple y Tratamiento Rápido

TCC: Temperatura Corporal Central

\section{Referencias Bibliográficas}

1. Aragall X. Refugiados e inmigrantes en el Mediterráneo [Internet]. Institut Europeu de la Mediterrània. 2015 [citado 8 de octubre de 2019]. Disponible en:

https://www.iemed.org/sala-de-premsa/iemed-als-mitjans/refugiados-e-inmigrantes-en-el-mediterraneo

2. 2016, récord de muertes en el mar Mediterráneo I eAcnur [Internet]. ACNUR. 2016 [citado 7 de octubre de 2019]. Disponible en:

https:/eacnur.org/es/actualidad/noticias/emergencias/2016-record-de-muertes-en-el-mar-mediterraneo

3. Más de 15.000 personas desaparecidas en el Mediterráneo | Agencia ONU Refugiados [Internet]. ACNUR. 2018 [citado 8 de octubre de 2019]. Disponible en:

https://eacnur.org/es/actualidad/noticias/emergencias/mas-de-15000-personas-desaparecidas-en-el-medit erraneo

4. ACNUR - Travesías desesperadas: refugiados e inmigrantes llegan a Europa y a las fronteras europeas [Internet]. 2018 [citado 7 de octubre de 2019]. Disponible en:

https://www.acnur.org/es-es/publications/pub_prot/5c5110f94/travesias-desesperadas-refugiados-e-inmig rantes-llegan-a-europa-y-a-las.html?query=refugiado mediterraneo 
5. Missing Migrants Project [Internet]. IOM. [citado 10 de octubre de 2019]. Disponible en: https://missingmigrants.iom.int/

6. OMI. Convención de las Naciones Unidas sobre el derecho del mar [Internet]. Organización Marítima Internacional. [citado 8 de octubre de 2019]. Disponible en: http://www.imo.org/es/OurWork/Legal/Paginas/UnitedNationsConventionOnTheLawOfTheSea.aspx

7. Lista de enmiendas al Capítulo V del SOLAS 74/88 [Internet]. [citado 8 de octubre de 2019]. Disponible en: https://www.fomento.es/recursos_mfom/pdf/3AE70A73-89B9-4A0B-8F46-E59F7511419A/121750/SOLASC APITULOVSEGURIDADENLANAVEGACION.pdf

8. Rodrigo de Larrueca J. El salvamento marítimo y el derecho humanitario [Internet]. 2018 [citado 8 de octubre de 2019]. Disponible en: https://upcommons.upc.edu/bitstream/handle/2117/120260/363n_OMI_Esp_270718_Rev_03).pdf

9. Fernández Sánchez PA. Migrantes, refugiados y víctimas del tráfico de personas en el Mediterráneo (entre la política y los derechos humanos)). Rev IUS [Internet]. 2017 [citado 7 de octubre de 2019];11(40):105-35. Disponible en: http://www.scielo.org.mx/scielo.php?pid=S1870-21472017000200105\&script=sci_arttext\&tlng=en

10. Mediterráneo: la ruta migratoria más letal del mundo | Médicos Sin Fronteras [Internet]. Médicos Sin Fronteras. 2019 [citado 9 de octubre de 2019]. Disponible en: https://www.msf.es/actualidad/mediterraneo/mediterraneo-la-ruta-migratoria-mas-letal-del-mundo

11. Oroza Busutil R, Puente Márquez Y. La crisis migratoria en el Mediterráneo y la Unión Europea: principales políticas y medidas antiinmigrantes. Rev Noved en Población [Internet]. 2017 [citado 9 de octubre de 2019];13(26):1-9. Disponible en: http://scielo.sld.cu/scielo.php?script=sci_arttext\&pid=S1817-40782017000200001

12. Acción Humanitaria: Principios [Internet]. Diccionario de Acción Humanitaria y Cooperación al Desarrollo. [citado 9 de octubre de 2019]. Disponible en: http://www.dicc.hegoa.ehu.es/listar/mostrar/4

13. Misiones Open Arms [Internet]. Open Arms. Sea Rescue. [citado 9 de octubre de 2019]. Disponible en: https://www.openarms.es/es/misiones

14. Open Arms. Quienes somos [Internet]. 2017 [citado 20 de octubre de 2019]. p. 1-29. Disponible en: https://www.openarms.es/uploads/quienes_somos/MEMO_2017_CASTELLANO.pdf

15. SOS Mediterranée [Internet]. [citado 8 de octubre de 2019]. Disponible en: https://sosmediterranee.org/

16. A la deriva en el Mediterráneo [Internet]. Amnistía Internacional. 2019 [citado 8 de octubre de 2019]. Disponible en: https://www.es.amnesty.org/en-que-estamos/reportajes/a-la-deriva-mediterraneo/

17. Preguntas frecuentes sobre salud y migración [Internet]. OMS. World Health Organization; 2017 [citado 10 de octubre de 2019]. Disponible en: https:/www.who.int/features/qa/88/es/

18. Avellanas M.L., Ricart A, Botella J, Mengelle F, Soteras I, Veres T, et al. Manejo de la hipotermia accidental severa. Medicina Intensiva [Internet]. 2012 [citado 11 de octubre de 2019];36(3):200-12. Disponible en: http://scielo.isciii.es/scielo.php?pid=S0210-56912012000300006\&script=sci_arttext\&tlng=pt

19. Romero Gónzalez RC. Triage en Emergencias Extrahospitalarias. Paraninfo Digit [Internet]. 2014 [citado 17 de octubre de 2019];20. Disponible en: http://www.index-f.com/para/n20/pdf/095.pdf

20. Cuartas Álvarez T, Castro Delgado R, Arcos González PI. Aplicabilidad de los sistemas de triaje prehospitalarios en los incidentes de múltiples víctimas. Emergencias: Revista de la Sociedad Española de Medicina de Urgencias y Emergencias, ISSN 1137-6821, Vol 26, No 2 (Abril), 2014, págs 147-154 [Internet]. 2014 [citado 17 de octubre de 2019];26(2):147-54. Disponible en: https://dialnet.unirioja.es/servlet/articulo?codigo=5427696

21. Szpilman D. MANUAL EMERGENCIAS ACUATICAS [Internet]. 2015 [citado 29 de octubre de 2019]. Disponible en: http://www.sobrasa.org/new_sobrasa/arquivos/baixar/Manual de emergencias acuaticas SOBRASA ESP.pdf

22. Ferrandini Price M, Arcos González PI, Pardo Ríos M, Nieto Fernández Pacheco A, Cuartas Alvarez T, Castro Delgado R. Comparación de los sistemas de triaje META y START en un ejercicio simulado de múltiples víctimas. Emergencias: Revista de la Sociedad Española de Medicina de Urgencias y Emergencias [Internet]. agosto de 2018 [citado 17 de octubre de 2019];30(4):224-30. Disponible en: https://dialnet.unirioja.es/servlet/articulo?codigo=6489694

23. Hart A, Nammour E, Mangolds V, Broach J. Intuitive versus Algorithmic Triage. Prehosp Disaster Med [Internet]. 21 de agosto de 2018 [citado 17 de octubre de 2019];33(4):355-61. Disponible en: http://www.ncbi.nlm.nih.gov/pubmed/30129913 
24. Jain T, Sibley A, Stryhn H, Hubloue I. Comparison of unmanned aerial vehicle technology versus standard practice in triaging casualties by paramedic students in a mass casualty incident scenario. CJEM [Internet]. 11 de mayo de 2018 [citado 17 de octubre de 2019];20(1):S83-S83. Disponible en: https://www.cochranelibrary.com/central/doi/10.1002/central/CN-01612204/full

25. Salvamento Marítimo. Informe anual 2018 [Internet]. 2018 [citado 5 de noviembre de 2019]. Disponible en: http://www.salvamentomaritimo.es/statics/multimedia/documents/2019/09/30/memoria_salvamento_201 8_23sept.pdf

26. Campos Chacón N. Hipotermia: a propósito de un caso. Medicina Legal de Costa Rica [Internet]. diciembre de 2016 [citado 11 de octubre de 2019];33(2):159-64. Disponible en: https://www.scielo.sa.cr/scielo.php?script=sci_arttext\&pid=S1409-00152016000200159

27. Paal P, Gordon L, Strapazzon G, Brodmann Maeder M, Putzer G, Walpoth B, et al. Accidental hypothermia-an update : The content of this review is endorsed by the International Commission for Mountain Emergency Medicine (ICAR MEDCOM). Scand J Trauma Resusc Emerg Med [Internet]. 2016 [citado 18 de octubre de 2019];24:111-31. Disponible en:

https://www.ncbi.nlm.nih.gov/pubmed/?term=Accidental+hypothermia-an+update \%3A+The+content+of +this+review+is+endorsed+by+the+International+Commission+for+Mountain

28. Strapazzon G, Reisten O, Argenone F, Zafren K, Zen-Ruffinen G, Larsen GL, et al. International Commission for Mountain Emergency Medicine Consensus Guidelines for On-Site Management and Transport of Patients in Canyoning Incidents. Wilderness Environ Med [Internet]. 2018 [citado 18 de octubre de 2019];29(2):252-65. Disponible en: http://www.ncbi.nlm.nih.gov/pubmed/29422373

29. Duong H, Patel G. Hypothermia . 2019. StatPearls [citado 19 de octubre de 2019]. Disponible en: https://www.ncbi.nlm.nih.gov/books/NBK545239/

30. Rischall ML, Rowland-Fisher A. Evidence-Based Management Of Accidental Hypothermia In The Emergency Department [Internet]. Emergency medicine practice. 2016 [citado 18 de octubre de 2019]; 18(1):1-18. Disponible en: https://www.ncbi.nlm.nih.gov/pubmed/26655247

31. Dow J, Giesbrecht GG, Danzl DF, Brugger H, Sagalyn EB, Walpoth B, et al. Wilderness Medical Society Clinical Practice Guidelines for the Out-of-Hospital Evaluation and Treatment of Accidental Hypothermia: 2019 Update. Wilderness Environ Med [Internet]. noviembre de 2019 [citado 10 de diciembre de 2019]; 30(4):47-69. Disponible en: https://www.ncbi.nlm.nih.gov/pubmed/31740369

32. Ministerio de trabajo, migraciones y seguridad social. Guía Sanitaria a bordo [Internet]. 2017 [citado 2 de noviembre de 2019]. Disponible en:

http://www.seg-social.es/wps/portal/wss/internet/Trabajadores/TrabajadoresMar/35300/35399

33. Zafren K. Out-of-Hospital Evaluation and Treatment of Accidental Hypothermia. Emerg Med Clin North Am [Internet]. mayo de 2017 [citado 18 de octubre de 2019];35(2):261-79. Disponible en:

http://www.ncbi.nlm.nih.gov/pubmed/28411927

34. Cardiac Arrest in Accidental Hypothermia - ECC Guidelines [Internet]. AHA. 2010 [citado 20 de octubre de 2019]. Disponible en:

https://eccguidelines.heart.org/circulation/cpr-ecc-guidelines/part-10-special-circumstances-of-resuscitati on/cardiac-arrest-in-accidental-hypothermia/

35. Truhlář A, Deakin CD, Soar J, Eldin G, Khalifa A, Alfonzo A, et al. European Resuscitation Council Guidelines for Resuscitation 2015 Section 4. Cardiac arrest in special circumstances on behalf of the Cardiac arrest in special circumstances section Collaborators 1. Resuscitation [Internet]. 2015 [citado 31 de octubre de 2019];95(95):148-201. Disponible en: https://www.sciencedirect.com/science/article/pii/S0300957215003299?via\%3Dihub

36. Paal P, Brugger H, Strapazzon G. Accidental hypothermia. En: Handbook of clinical neurology [Internet]. 2018 [citado 16 de octubre de 2019]. 157:547-563. Disponible en: http://www.ncbi.nlm.nih.gov/pubmed/30459025

37. Mwaura L, Rubino A, Vuylsteke A. No Cold Death-Extracorporeal Life Support for All Victims of Accidental Hypothermia. J Cardiothorac Vasc Anesth [Internet]. 13 de septiembre de 2019 [citado 19 de octubre de 2019]; Disponible en: http://www.ncbi.nlm.nih.gov/pubmed/31587930

38. Foggle JL. Accidental Hypothermia: «You're Not Dead Until You're Warm and Dead». R I Med J (2013) [Internet]. 1 de febrero de 2019 [citado 20 de octubre de 2019];102(1):28-32. Disponible en: http://www.ncbi.nlm.nih.gov/pubmed/30709071 
39. Saczkowski RS, Brown DJA, Abu-Laban RB, Fradet G, Schulze CJ, Kuzak ND. Prediction and risk stratification of survival in accidental hypothermia requiring extracorporeal life support: An individual patient data meta-analysis. Resuscitation [Internet]. junio de 2018 [citado 19 de octubre de 2019];127:51-7. Disponible en: http://www.ncbi.nlm.nih.gov/pubmed/29580960

40. Kieboom JK, Verkade HJ, Burgerhof JG, Bierens JJ, Van Rheenen PF, Kneyber MC, et al. Outcome after resuscitation beyond 30 minutes in drowned children with cardiac arrest and hypothermia: Dutch nationwide retrospective cohort study. BMJ [Internet]. 10 de febrero de 2015 [citado 19 de octubre de 2019];350. Disponible en: http://www.ncbi.nlm.nih.gov/pmc/articles/PMC4353310/

41. Kriz D, Piantino J, Fields D, Williams C. Pediatric Hypothermic Submersion Injury and Protective Factors Associated with Optimal Outcome: A Case Report and Literature Review. Children [Internet]. 27 de diciembre de 2017 [citado 19 de octubre de 2019];5(1):4. Disponible en: https://www.https//www.ncbi.nlm.gov/pmc/articles/PMC5789286/

42. Avellanas Chavala ML, Ayala Gallardo M, Soteras Martínez, Subirats Bayego E. Management of accidental hypothermia: A narrative review. Med Intensiva [Internet]. 2019 [citado 19 de octubre de 2019]; 43(9):556-568. Disponible en: https://www.ncbi.nlm.nih.gov/pubmed/30683520

43. Haverkamp FJC, Giesbrecht GG, Tan ECTH. The prehospital management of hypothermia - An up-to-date overview. Injury [Internet]. febrero de 2018 [citado 18 de octubre de 2019];49(2):149-64. Disponible en: http://www.ncbi.nlm.nih.gov/pubmed/29162267

44. Freeman S, Deakin CD, Nelson MJ, Bootland D. Managing accidental hypothermia: a UK-wide survey of prehospital and search and rescue providers. Emerg Med J [Internet]. noviembre de 2018 [citado 18 de octubre de 2019];35(11):652-6. Disponible en: http://www.ncbi.nlm.nih.gov/pubmed/30026185

45. Burggraf M, Lendemans S, Waack IN, Teloh JK, Effenberger-Neidnicht K, Jäger M, et al. Slow as Compared to Rapid Rewarming After Mild Hypothermia Improves Survival in Experimental Shock. J Surg Res [Internet]. abril de 2019 [citado 19 de octubre de 2019];236:300-10. Disponible en: http://www.ncbi.nlm.nih.gov/pubmed/30694770

46. Greif R, Rajek A, Laciny S, Bastanmehr H, Sessler DI. Resistive heating is more effective than metallic-foil insulation in an experimental model of accidental hypothermia: A randomized controlled trial. Ann Emerg Med [Internet]. 2000 [citado 19 de octubre de 2019];35(4):337-45. Disponible en: https://www.ncbi.nlm.nih.gov/pubmed/10736119

47. Giesbrecht GG, Walpoth BH. Risk of Burns During Active External Rewarming for Accidental Hypothermia. Wilderness Environ Med [Internet]. 28 de septiembre de 2019 [citado 19 de octubre de 2019];30(4):431-436. Disponible en: http://www.ncbi.nlm.nih.gov/pubmed/31575480

48. Kumar P, McDonald GK, Chitkara R, Steinman AM, Gardiner PF, Giesbrecht GG. Comparison of Distal Limb Warming With Fluidotherapy and Warm Water Immersion for Mild Hypothermia Rewarming. Wilderness Environ Med [Internet]. septiembre de 2015 [citado 19 de octubre de 2019];26(3):406-11. Disponible en: http://www.ncbi.nlm.nih.gov/pubmed/26116985

49. Van der Ploeg G-J, Goslings JC, Walpoth BH, Bierens JJLM. Accidental hypothermia: rewarming treatments, complications and outcomes from one university medical centre. Resuscitation [Internet]. noviembre de 2010 [citado 19 de octubre de 2019];81(11):1550-5. Disponible en: http://www.ncbi.nlm.nih.gov/pubmed/20702016

50. Hou Y, Qiao Y, Xiong M, Zhang D, Rao W, Shi C. Hypothermia-rewarming: A Double-edged sword? Med Hypotheses [Internet]. 30 de agosto de 2019 [citado 19 de octubre de 2019];133:109387. Disponible en: http://www.ncbi.nlm.nih.gov/pubmed/31541781

51. Christensen ML, Lipman GS, Grahn DA, Shea KM, Einhorn J, Heller HC. A Novel Cooling Method and Comparison of Active Rewarming of Mildly Hypothermic Subjects. Wilderness Environ Med [Internet]. junio de 2017 [citado 19 de octubre de 2019];28(2):108-15. Disponible en: http://www.ncbi.nlm.nih.gov/pubmed/28506514

52. Liu X, Shi Y, Ren C, Li X, Zhang Z. Effect of an electric blanket plus a forced-air warming system for children with postoperative hypothermia: a randomized controlled trial I Cochrane Library. 2017 [citado 19 de octubre de 2019];96(26). Disponible en: https://www.cochranelibrary.com/central/doi/10.1002/central/CN-01381421/full

53. Yang H, Lee H, Chu T, Su Y, Ho L, Fan J. The comparison of two recovery room warming methods for hypothermia patients who had undergone spinal surgery | Cochrane Library. 2012 [citado 19 de octubre 
de 2019];44(1):2-10. Disponible en:

https://www.cochranelibrary.com/central/doi/10.1002/central/CN-01933693/full

54. Peters H, Bednarczyk J, White C, Weldon E, Singal R. Severe accidental hypothermia treated with extra-corporeal membrane oxygenation in an urban Canadian setting | Cochrane Library. 2017 [citado 19 de octubre de 2019];(6). Disponible en:

https://www.cochranelibrary.com/central/doi/10.1002/central/CN-01375681/full

55. Zhang R, Chen X, Xiao Y. The effects of a forced-air warming system plus electric blanket for elderly patients undergoing transurethral resection of the prostate: a randomized controlled trial I Cochrane Library. 2018 [citado 19 de octubre de 2019];97(45). Disponible en:

https://www.cochranelibrary.com/central/doi/10.1002/central/CN-01664490/full

(C) 2020 por los autores; Esta obra está sujeta a la licencia de Reconocimiento 4.0

Internacional de Creative Commons. Para ver una copia de esta licencia, visite http://creativecommons.org/licenses/by-nc-nd/4.0/. 